\title{
Online network of subspecialty aortic disease experts: Impact of "cloud" technology on management of acute aortic emergencies
}

\author{
Paul Schoenhagen, MD, ${ }^{\mathrm{a}, \mathrm{b}}$ Eric E. Roselli, MD,${ }^{\mathrm{c}}$ C. Martin Harris, MD, ${ }^{\mathrm{d}}$ Matthew Eagleton, MD, ${ }^{\mathrm{e}}$ and \\ Venu Menon, MD $^{\mathrm{a}}$
}

\begin{abstract}
For the management of acute aortic syndromes, regional treatment networks have been established to coordinate diagnosis and treatment between local emergency rooms and central specialized centers. Triage of acute aortic syndromes requires definitive imaging, resulting in complex data files. Modern information technology network structures, specifically "cloud" technology, coupled with mobile communication, increasingly support sharing of these data in a network of experts using mobile, online access and communication. Although this network is technically complex, the potential benefit of online sharing of data files between professionals at multiple locations within a treatment network appear obvious; however, clinical experience is limited, and further evaluation is needed. (J Thorac Cardiovasc Surg 2016;152:39-42)
\end{abstract}

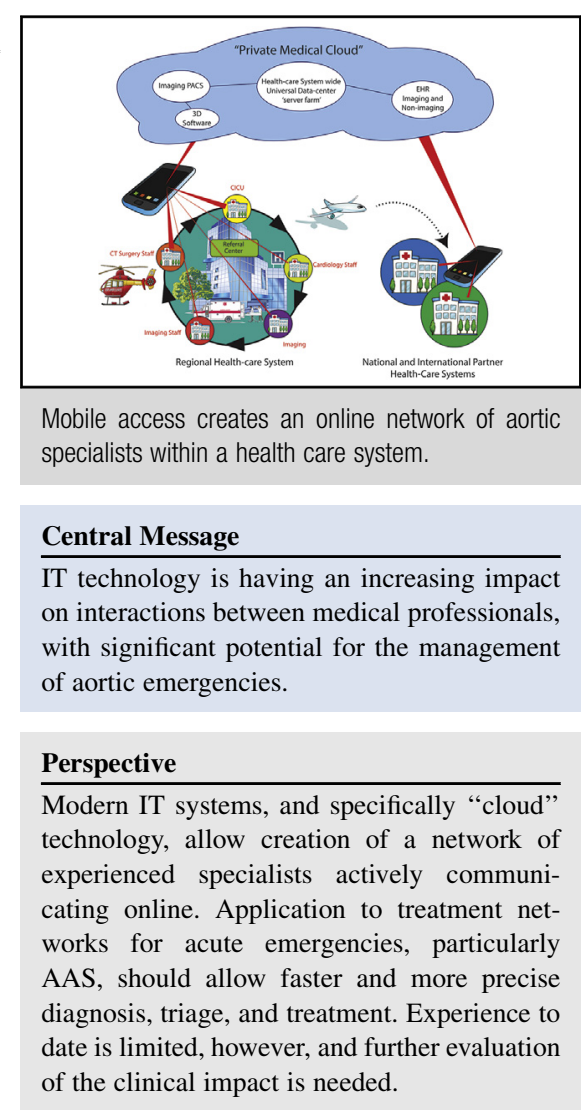

See Editorial Commentary page 42.
Modern information technology (IT), and specifically the widespread distribution of handheld devices including smartphones, has changed many aspects of communication and social interaction. In the medical field, IT systems have transformed health care delivery, supporting completely digital electronic health records (EHRs), wireless transmission of data, and mobile communication. Although cell phone

From the Departments of ${ }^{\mathrm{a}}$ Cardiovascular Medicine, ${ }^{\mathrm{c}}$ Thoracic and Cardiovascular Surgery, ${ }^{\mathrm{d}}$ Internal Medicine, and ${ }^{\mathrm{e}}$ Vascular Surgery, and ${ }^{\mathrm{b}}$ Imaging Institute, Cleveland Clinic, Cleveland, Ohio.

Received for publication Jan 18, 2016; revisions received Feb 22, 2016; accepted for publication Feb 26, 2016; available ahead of print March 22, 2016.

Address for reprints: Paul Schoenhagen, MD, Cardiovascular Imaging, Desk J1-4, Cleveland Clinic, 9500 Euclid Ave, Cleveland, OH 44195 (E-mail: schoenp1@ ccf.org).

$0022-5223 / \$ 36.00$

Copyright (c) 2016 by The American Association for Thoracic Surgery http://dx.doi.org/10.1016/j.jtcvs.2016.02.057 use was prohibited in hospitals just a few years ago, it is now becoming an integral part of the interaction among medical professionals, allowing exchanges of complex data using mobile devices. A particularly relevant example is the management of medical emergencies, including acute myocardial infarction and acute aortic syndromes (AAS). For these emergencies, regional treatment networks have been established to coordinate diagnosis, triage, and treatment between local emergency rooms as the initial point of contact and central specialized centers experienced in definitive pharmacologic, interventional, or surgical treatment. ${ }^{1-7}$

Within these regional treatment networks, the availability of subspecialty experts (eg, cardiology, cardiothoracic and vascular surgery, intensive care medicine, anesthesiology, radiology) is critical for interpreting and performing timesensitive diagnostic and therapeutic interventions. For conditions with a higher incidence (ie, one or several per day), 


\section{Abbreviations and Acronyms \\ AAS = acute aortic syndromes \\ EHR $=$ electronic health record \\ IT $=$ information technology \\ PACS $=$ picture archiving and communication system}

as is the case for acute ST elevation myocardial infarction, $24 / 7$ availability of experts within the central (hub) hospital is feasible and easily achieved. For less frequent conditions such as AAS, rapid response teams are available 24/7 to provide consistent care, but the team is less often directly available within the hospital. Instead, a network of specialists remotely directs the initial diagnosis and triage of patients in a larger geographic area.

In contrast to acute myocardial infarction, for which electrocardiography and serum markers are critical for early decision making, AAS lacks simple diagnostic biomarkers. Triage of patients with suspected AAS requires definitive imaging. Computed tomography is typically performed and interpreted at the initial point of access in the local emergency department. Subspecialty expertise is often lacking after the initial diagnosis, however, and thus false-positive transfer activation is not uncommon, especially in the setting of complex previous endovascular repair. Large image data files have traditionally been transferred with the patient either in film or CD format. At the tertiary referral center, the images are then downloaded and subsequently reinterpreted to form the basis for definitive treatment. Proactive triage before or during patient transfer would require an early, remote review by specialists available within the network and communication among professionals at different locations. ${ }^{8}$

Modern IT network structures, and specifically "cloud" technology, coupled with mobile communication, increasingly support such an approach (Figure 1). These systems allow the uploading of data from multiple sites onto central cloud servers and subsequent access and review from anywhere within the network. ${ }^{9,10}$ Mobile online access to the central EHR, including imaging and nonimaging data from tablet computers and smartphones, allows review without limitations of place and time, and enables surveillance and online communication within a network of specialists. Optimally, data are organized as a comprehensive patientcentered integrated EHR file, including history and physical examination results, laboratory and imaging data, and corresponding reports. In large health care systems, this requires a complex infrastructure/network spanning multiple locations. A central data center connects a large number of servers (a server farm) with integration among the EHR, a picture archiving and communication system (PACS), and additional 3D/4D analysis tools. The core storage center provides access to all data generated in the healthcare system, which can be visualized in a patient-centric fashion through the electronic medical record, PACS, or a more dedicated system. Access at multiple locations is achieved by client server solutions, where a single dataset is maintained on the central server, whereas the local workstation serves only as the access point. These systems are a form of cloud computing, described as a "private medical-grade cloud." Hospital systems may be able to maintain a private cloud or, alternatively, use a third-party data service, but issues of reliable patient identification and data security need to be clarified.

Although such a network is technically complex, the benefit of online sharing of data files among professionals at multiple locations within a treatment network appears obvious. ${ }^{9,10}$ Clinical experience to date is limited, however. The time-sensitive nature and relatively low incidence of AAS, the complexity of open or endovascular surgery, and the relative paucity of institutions that deliver 24/7 state-of-the-art care have been cited as the clinical rationale for the creation of regional AAS treatment networks. The safety and timeliness of patient transfer provided within regional aortic networks has been reported. ${ }^{3,11,12}$ In an investigation of the prevalence and etiology of falsepositive diagnoses in patients transferred to a tertiary referral center for suspected AAS, our group found falsepositive suspicion of dissection in 17 of $150(11.3 \%)$ consecutive patients transferred for suspected AAS from community emergency departments directly to the cardiac intensive care unit. ${ }^{11,12}$ False-positive activation was driven primarily by uncertainty secondary to motion artifact of the ascending aorta and the presence of complex anatomy after previous aortic intervention. ${ }^{13}$ Network-wide standardization of imaging strategies, along with improved sharing of imaging with early expert review, may further improve triage of this complex patient population.

Our group has further evaluated the diagnostic accuracy of hand-held devices compared with conventional dedicated workstations for diagnosing acute aortic pathology. ${ }^{14}$ In 104 patients who underwent a computed tomography scan during on-call hours for suspected AAS, assessment was performed on a hand-held device connected via a secure connection to Web-based PACS servers. The subsequent interpretation from a dedicated workstation coupled with the diagnosis at the time of discharge served as the reference standard for determining the presence or absence of an acute abnormality. Evaluation with the hand-held devices demonstrated good sensitivity and specificity, suggesting that hand-held devices can be a potential useful tool in the diagnosis and triage of patients presenting with cardiovascular emergencies.

Understanding the morphological details of the disease process also can help expedite care after arrival. Remote data upload on a central server initiated at the initial point of access, and image review before or at the time of transfer, may allow the treating surgeon to determine whether a 


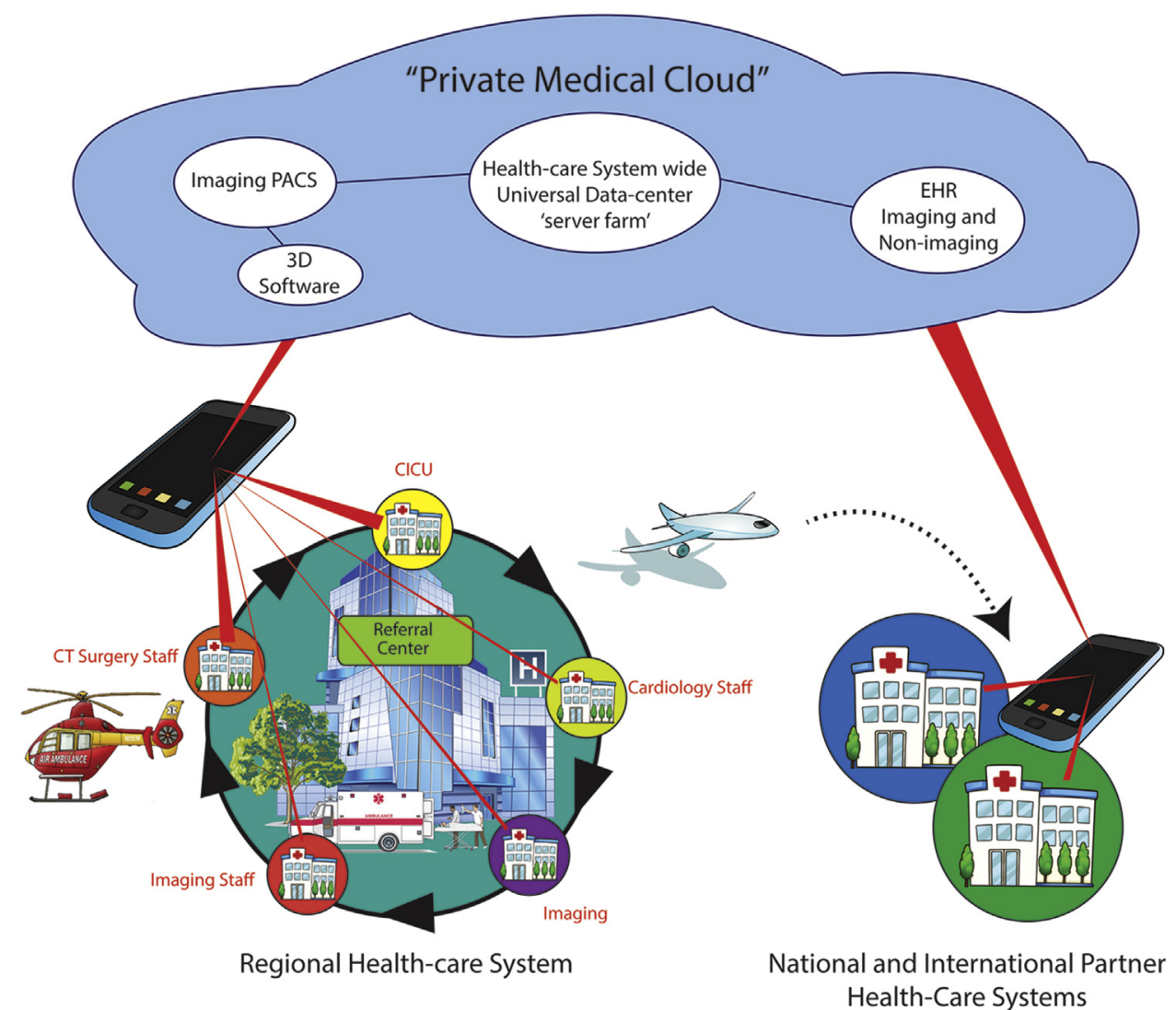

FIGURE 1. The IT structure ("private medical cloud") allowing online, mobile access and communication within a regional health care system and its national and international partners. Subspecialists in the tertiary referral center are connected via an online network to one another and to colleagues in the regional health care system, allowing coordination of care. PACS, Picture archiving and communication system; $E H R$, electronic health record; $C I C U$, cardiac intensive care unit; $C T$, cardiothoracic.

patient will require an open, endovascular, or hybrid approach to surgical intervention. We are currently evaluating the integration of EHR and imaging data in a single mobile application, along with proactive communication within a group of aortic disease specialists. This information and communication may impact the activation of appropriate team members, preparation of devices, and coordination of nursing care.

In summary, modern IT technology is having an increasing impact on interaction among medical professionals, with a significant disruptive potential for traditional on-call structures in the management of acute emergencies. Modern cloud technology allows the creation of networks of experienced specialists actively communicating online. Application to treatment networks for acute emergencies, particularly AAS, should allow for faster and more precise diagnosis, triage, and subsequent treatment. Experience to date is limited, however, and further evaluation of the appropriate, secure infrastructure required for reliable patient identification between provider organizations, definition of experts' qualifications, and standardization of treatment algorithms is needed. Eventually, collection and evaluation of original data describing potential clinical impact will be critical.

\section{Conflict of Interest Statement}

E.R. has received personal fees from Vascutek, Medtronic, Bolton Medical, Cook Medical, and Gore Medical outside the submitted work. M.E. has received personal fees from Bolton Medical and Cook Medical outside the submitted work. All other authors have nothing to disclose with regard to commercial support.

\section{References}

1. Boyd DR, Dunea MM, Flashner BA. The Illinois plan for a statewide system of trauma centers. J Trauma. 1973;13:24-31.

2. Cowley RA, Hudson F, Scanlan E, Gill W, Lally RJ, Long W, et al. An economical and proved helicopter program for transporting the emergency critically ill and injured patient in Maryland. J Trauma. 1973;13:1029-38.

3. Harris KM, Strauss CE, Duval S, Unger BT, Kroshus TJ, Inampudi S, et al. Multidisciplinary standardized care for acute aortic dissection: design and initial outcomes of a regional care model. Circ Cardiovasc Qual Outcomes. 2010;3: 424-30.

4. Henry TD, Sharkey SW, Burke MN, Chavez IJ, Graham KJ, Henry CR, et al. A regional system to provide timely access to percutaneous coronary intervention for ST-elevation myocardial infarction. Circulation. 2007;116:721-8. 
5. Ting HH, Rihal CS, Gersh BJ, Haro LH, Bjerke CM, Lennon RJ, et al. Regional systems of care to optimize timeliness of reperfusion therapy for ST-elevation myocardial infarction: the Mayo Clinic STEMI Protocol. Circulation. 2007; 116:729-36.

6. Jollis JG, Roettig ML, Aluko AO, Anstrom KJ, Applegate RJ, Babb JD, et al. Implementation of a statewide system for coronary reperfusion for ST-segment elevation myocardial infarction. JAMA. 2007;298:2371-80.

7. LaMonte MP, Bahouth MN, Magder LS, Alcorta RL, Bass RR, Browne BJ, et al A regional system of stroke care provides thrombolytic outcomes comparable with the NINDS stroke trial. Ann Emerg Med. 2009;54:319-27.

8. Cowie MR, Chronaki CE, Vardas P. e-Health innovation: time for engagement with the cardiology community. Eur Heart J. 2013;34:1864-8.

9. Schoenhagen P, Zimmermann M, Falkner J. Advanced 3-D analysis, clientserver systems, and cloud computing: integration of cardiovascular imaging data into clinical workflows of transcatheter aortic valve replacement. Cardiovasc Diagn Ther. 2013;3:80-92.

10. Schoenhagen P, Falkner J, Piraino D. Transcatheter aortic valve repair, imaging, and electronic imaging health record. Curr Cardiol Rep. 2013;15:319.
11. Aggarwal B, Raymond C, Jacob J, Kralovic D, Kormos K, Holloway D, et al. Transfer of patients with suspected acute aortic syndrome. Am J Cardiol. 2013;112:430-5.

12. Aggarwal B, Raymond CE, Randhawa MS, Roselli E, Jacob J, Eagleton M, et al. Transfer metrics in patients with suspected acute aortic syndrome. Circ Cardiovasc Qual Outcomes. 2014;7:780-2.

13. Raymond CE, Aggarwal B, Schoenhagen P, Kralovic DM, Kormos K, Holloway D, et al. Prevalence and factors associated with false-positive suspicion of acute aortic syndrome: experience in a patient population transferred to a specialized aortic treatment center. Cardiovasc Diagn Ther. 2013;3:196-204.

14. Matar R, Renapurkar R, Obuchowski N, Menon V, Piraino D, Schoenhagen P. Utility of hand-held devices in diagnosis and triage of cardiovascular emergencies: observations during implementation of a PACS-based system in an acute aortic syndrome (AAS) network. J Cardiovasc Comput Tomogr. 2015;9:524-33.

Key Words: acute aortic syndrome, aortic dissection, imaging, private medical cloud, disease management networks

\title{
EDITORIAL COMMENTARY
}

\section{Are we ready to sit on a little cloud?}

\author{
Jean Bachet, MD
}

From ADETEC, Suresnes, France.

Disclosures: Author has nothing to disclose with regard to commercial support

Received for publication Feb 29, 2016; accepted for publication March 1, 2016; available ahead of print March 31, 2016.

Address for reprints: Jean Bachet, MD, ADETEC, 1 Place Marcel Legras, 92150 Suresnes, France (E-mail: jean. bachet@yahoo.fr).

J Thorac Cardiovasc Surg 2016;152:42-3

$0022-5223 / \$ 36.00$

Copyright (c) 2016 by The American Association for Thoracic Surgery

http://dx.doi.org/10.1016/j.jtcvs.2016.03.018

The article by Schoenhagen and colleagues in this issue of the Journal proposes the organization of large and centralized information technology (IT) networks of "experts" to assess and codify the management of patients suffering from acute aortic syndromes. ${ }^{1}$ Obviously this idea finds its origin in and could be made possible through the easiness and strength of communication in the present times and the quality of information exchange that can be obtained through those network systems.

It is certainly a good idea that may represent a step toward a more evolved and reliable system for managing routine patient care and treating emergencies. As a matter of fact, the proposed network might represent significant progress in terms of diagnosis through, for example, better analysis and assessment of the provided images, of the patient's clinical condition, advice concerning additional explorations, and so on.

Yet it seems that such a system might have some drawbacks or, at least that its implementation might merit some caution. Indeed, it may be rather difficult to define an

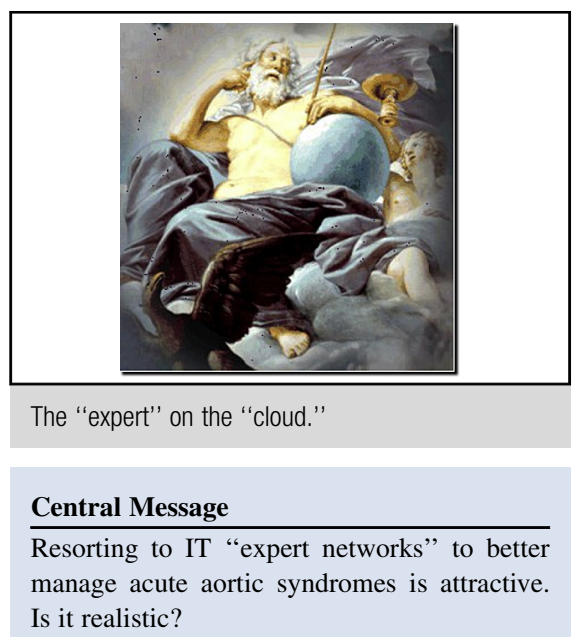

See Article page 39.

"expert." It is a subjective view, and the term may be applied to professionals with widely varying kinds or levels of skill and knowledge. The English Oxford Dictionary defines an "expert" as "a person who is very knowledgeable about or skilful in a particular area." But the definition is associated with the following example: "In every area of life, the experts are ready to smooth our path and tell us what to believe." Indeed. Expertise is not a condition based on simple, unquestionable, reproducible scientific criteria, but rather it is the complex 